\title{
EDITORIAL
}

\section{Frutas y verduras perdidas y desperdiciadas, una oportunidad para mejorar el consumo}

\author{
Carmen Gloria González G. \\ Nutricionista, MSc Nutrición Humana \\ Instituto de Nutrición y Tecnología de los Alimentos (INTA) Universidad de Chile \\ E-mail: carmen.gonzalez@inta.uchile.cl
}

Las pérdidas y desperdicios de alimentos (PDA) son una preocupación a nivel mundial, ya que se estima que cada año 1.300 toneladas de alimentos son eliminados estando en condiciones de ser consumidos por los seres humanos'. Los alimentos se pierden a nivel de producción, cosecha y post cosecha y se desperdician en todos los ámbitos del consumo (almacenamiento, distribución y uso en el hogar). Las PDA impactan la sostenibilidad de los sistemas alimentarios, reduciendo la disponibilidad y acceso a los alimentos y deteriorando el uso sostenible de los recursos naturales para las producciones futuras 2 .

Las frutas y verduras (FyV) son el grupo de alimentos con mayores pérdidas (hasta el $45 \%$ de lo producido) y también representan los mayores niveles de desperdicios (hasta el 30\% de las compras realizadas por los consumidores) ${ }^{3}$. Las razones por las que se produce su desecho son estéticas, es decir, por su forma, tamaño y calibre, que corresponden básicamente a los criterios de selección para su venta. Por otro lado, partes de las FyV, no son valorizadas (los tallos, hojas, cáscaras), ya que no son el componente principal o de preferencia de las FyV y son eliminados sin considerar su aporte nutricional.

La Organización Mundial de la Salud recomienda un consumo de FyV de al menos 400 gramos al día por persona (5 porciones/día; $80 \mathrm{gr} /$ porción) para acceder a los múltiples beneficios de su consumo, incluido la prevención de enfermedades ${ }^{4}$. Por ejemplo, para la disminución del riesgo del desarrollo de cáncer, se ha establecido un consumo recomendado de 600 gramos al día de FyV 5 . Incluso se obtendrían mayores beneficios en la prevención de enfermedades, consumiendo 800 gramos al día de FyV, pudiendo evitarse hasta 7,8 millones de muertes prematuras a nivel mundial ${ }^{6}$. Aunque las enfermedades crónicas no transmisibles y la obesidad continúan aumentado a nivel global7, el consumo de FyV sigue siendo bajo. La Encuesta Nacional de Salud de Chile 2016-2017, mostró que el consumo de FyV promedio fue de 178 gramos/persona/día, menos de la mitad de la recomendación y que solo el $15 \%$ de los Chilenos consume 5 porciones diarias de FyV 8 .

En la actualidad, se realizan diversos esfuerzos para promover el consumo de FyV. Entre ellos, el Programa 5 al día Chile, fomenta el consumo de estos alimentos desde el año 2006, contando con el apoyo del Ministerio de Agricultura, la FAO y OPS y el sector privado ${ }^{9}$. Sin embargo, se requieren cambios para lograr aumentar el consumo de FyV en forma efectiva. La educación alimentaria es fundamental para mejorar los conocimientos y habilidades de las personas, en la selección y preparación de estos alimentos y se necesitan modificaciones importantes en los ambientes alimentarios, que permitan aumentar la disponibilidad y oferta de FyV. En este sentido, el rescate de las FyV que son perdidas y desperdiciadas en diversos momentos, puede complementar la ingesta insuficiente. Aquellos alimentos que se eliminan por no contar con las características estéticas que el mercado exige, podrían ser dispuestas para mejorar la nutrición de las personas más vulnerables, a través de donaciones. También, se podrían vender a precios más económicos, derribando la barrera de los costos, como razón para no comprarlos. A nivel del consumidor, el almacenamiento y manipulación adecuada y la utilización de partes aprovechables que se desperdician, son una forma concreta de lograr aumentar el consumo de FyV en los hogares ${ }^{10}$. Por estas razones, visibilizar el problema de las PDA y educar en las acciones para prevenirlas a distintos niveles, constituyen una oportunidad para mejorar el consumo de FyV.

BIBLIOGRAFÍA

1. FAO. Global food losses and food waste-extent, causes and prevention. En Gustavsson J, Cederberg C, Sonesson U, van Otterdijk R, Meybeck A. Rome. 2011

2. FAO. Reflexiones sobre el sistema alimentario en América Latina y el Caribe y perspectivas para su sostenibilidad. Santiago de Chile. 2017

3. Save Food. Iniciativa Global sobre la Reducción de Pérdidas y Desperdicios de Alimentos, 2015

4. World Health Organization. Diet, nutrition and the prevention of chronic diseases. Report of the joint WHO/FAO expert consultation WHO Technical Report Series, No. 916. 2003

5. World Cancer Research Fund, American Institute for Cancer Research. Food, Nutrition, Physical Activity, and the Prevention of Cancer: a Global Perspective. Washington, DC: World Cancer Research Fund International; 2007

6. Aune D, Giovannucci E, Boffetta P, Fadnes L, Keum N, Norat T, Greenwood D, Riboli E, Vatten L, Tonstad S. 10 Fruit and vegetable intake and the risk of cardiovascular disease, total cancer and all-cause mortality. A systematic review and dose response meta-analysis of prospective studies. Int J Epidemiol. 2017; 46(3): 1029-1056

7. The GBD 2015 Obesity Collaborators Health Effects of Overweight and Obesity in 195 Countries over 25 Years. N Engl J Med 2017; $377: 13-27$

8. Ministerio de Salud de Chile. Encuesta Nacional de Salud. 2016-2017

9. Zacarías I, González CG, Fonseca L, Domper A, Lera L, Vio F. Programa 5 al día Chile. A 10 años de su implementación. En: Realidad y perspectivas de la Producción y Consumo de verduras y frutas en Chile. Ministerio de Agricultura, Corporación 5 al día, INTA Universidad de Chile. Santiago Chile; 2016

10. Acuña D, Domper A, Eguillor P, González CG, Zacarías I, Manual de Pérdidas y Desperdicios de Alimentos. Santiago: Corporación 5 al día; Ministerio de Agricultura; INTA Universidad de Chile; 2018 